\title{
Lycopene attenuates inflammation and apoptosis in post-myocardial infarction remodeling by inhibiting the nuclear factor- $\kappa B$ signaling pathway
}

\author{
QIN HE, WEI ZHOU, CAIJIN XIONG, GANG TAN and MANHUA CHEN \\ Department of Cardiology, The Central Hospital of Wuhan, Wuhan, Hubei 430014, P.R. China
}

Received December 25, 2013; Accepted June 17, 2014

DOI: $10.3892 / \mathrm{mmr} .2014 .2676$

\begin{abstract}
Inflammatory response and cardiomyocyte apoptosis are important processes in ventricular remodeling post-myocardial infarction (MI) and may form the basic mechanisms in the development of chronic heart failure. The nuclear factor $\kappa \mathrm{B}(\mathrm{NF}-\kappa \mathrm{B})$ signaling pathway could promote inflammation and apoptosis and it has been demonstrated that lycopene inhibits cigarette smoke extract-mediated $\mathrm{NF}-\kappa \mathrm{B}$ activation. Therefore, it was hypothesized that the NF- $\mathrm{kB}$ signaling pathway may be a key target of lycopene in the reversal of ventricular remodeling post MI. An MI model was established by left anterior descending coronary artery ligation in mice. Following ligation, the mice were administered with lycopene $(10 \mathrm{mg} / \mathrm{kg} / \mathrm{day})$ or saline. The mice underwent echocardiography and were sacrificed after 4 weeks. The mRNA expression of fibrosis markers transforming growth factor- $\beta 1$ (TGF- $\beta 1$ ), collagen I and III and inflammatory markers tumor necrosis factor- $\alpha$ (TNF- $\alpha$ ) and interleukin-1 $\beta$ (IL-1 $\beta$ ) were examined by quantitative polymerase chain reaction. The protein expression of apoptotic markers, including caspase $-3,-8,-9$ and activation of the $\mathrm{NF}-\kappa \mathrm{B}$ signaling pathway were analyzed by western blotting. Lycopene reduced the expression of TGF- $\beta 1$, collagen I, collagen III, TNF- $\alpha$, IL- $1 \beta$, caspase- $-3,-8$ and -9 and inhibited the activation of the NF- $\mathrm{kB}$ signaling pathway. The level of ventricular remodeling post-MI was also attenuated following treatment with lycopene. Lycopene may inhibit the $\mathrm{NF}-\mathrm{\kappa B}$ signaling pathway thereby reducing the inflammatory response and cardiomyocyte apoptosis post-MI, which could be a key mechanism of lycopene in attenuating ventricular remodeling.
\end{abstract}

Correspondence to: Dr Manhua Chen or Miss Qin He, Department of Cardiology, The Central Hospital of Wuhan, 26 Shengli Street, Jiang'an, Wuhan, Hubei 430014, P.R. China

E-mail: chenmanhuawh@gmail.com

E-mail:ph610528@hotmail.com

Key words: myocardial infarction, ventricular remodeling, inflammatory response, apoptosis, lycopene

\section{Introduction}

Cardiac remodeling post myocardial infarction (MI) results in ventricular dysfunction and heart failure, which contribute to a poor outcome and high mortality rate $(1,2)$. Previous studies have reported that the inflammatory response and cardiomyocyte apoptosis were the underlying mechanisms in the development of chronic heart failure (3-5). MI causes local inflammation and apoptosis, which can result in cardiomyocyte damage (5). Proinflammatory mediators are upregulated in cardiac dysfunction and, in particular, elevated levels of inflammatory cytokines, including tumor necrosis factor- $\alpha$ (TNF- $\alpha$ ) and interleukin (IL)- $1 \beta$ in the infarcted myocardium contribute to cardiomyocyte apoptosis $(2,6,7)$. Cell apoptosis may also persist in infarcted areas $(8,9)$. The nuclear factor- $\mathrm{\kappa B}$ (NF- $\kappa \mathrm{B})$ signaling pathway, involved in inflammation, is also active in the apoptotic pathway. Several approaches to reduce the activation of NF- $\mathrm{kB}$ have been demonstrated to reduce myocardial inflammation and apoptosis (10-12). Therefore, inhibition of inflammation and apoptosis may be an effective therapeutic strategy for MI.

The NF- $\mathrm{kB}$ family is a key factor regulating the inflammatory response by promoting the expression of proinflammatory factors (13). A member of the NF-kB family, p65, forms homo or heterodimers that are bound to inhibitory (ІкB) proteins in the cytosol (14). Degradation of I $\mathrm{B}$ releases NF- $\kappa B$ dimers and enables the translocation of NF- $\mathrm{KB}$ into the nucleus, where it can initiate transcription of target genes. NF- $\mathrm{KB}$ activation and subsequent augmented inflammatory responses largely contribute to maladaptive left ventricular remodeling and cardiac dysfunction post-MI (15). Lycopene is a type of carotenoid, present predominantly in tomatoes. Several epidemiological studies have confirmed that high levels of plasma lycopene could reduce the risk of cardiovascular disease, neurodegenerative disease and cancer $(16,17)$. In addition, it was demonstrated that lycopene inhibited inflammatory cell infiltration and pathologic histological alterations following MI induced by isoprenaline $(18,19)$. A previous study also reported that lycopene inhibited the expression of interleukin (IL)-8 and the activation of NF- $\mathrm{KB}$ in the lungs induced by cigarette smoke (20) and reduced the expression of proinflammatory cytokines in adipose tissue of an animal model of obesity (21). However, the role and mechanism of lycopene in the inhibition of inflammation and apoptosis in MI are yet to be elucidated. 
In the present study, it was hypothesized that lycopene inhibited $\mathrm{NF}-\kappa \mathrm{B}$ activation, which mediates the inflammatory response and cardiomyocyte apoptosis in post-MI remodeling. Therefore, MI mice models were constructed by ligation of the left anterior descending coronary artery (LAD) to examine whether lycopene can inhibit $\mathrm{NF}-\kappa \mathrm{B}$ activation and thus reduce inflammation and apoptosis in the process of post-MI remodeling.

\section{Materials and methods}

Materials. Primary antibodies against NF- $\kappa \mathrm{B}, \mathrm{p} 65$ (cat no. 8242) and phospho-NF- $\mathrm{B}$ p65 (cat no. 3039) were purchased from Cell Signaling Technology, Inc. (Danvers, MA, USA). Primary antibodies against caspase-3, (cat no. ab4051) -8 (cat no. ab25901) and -9 (cat no. ab32539) were purchased from Abcam (Cambridge, MA, USA). Glyceraldehyde 3-phosphate dehydrogenase (GAPDH) was purchased from Bioworld Technology (St. Louis Park, MN, USA). The bicinchoninic acid protein assay kit was purchased from Pierce Biotechnology, Inc. (Rockford, IL, USA). TRIzol (cat no. 15596018) was purchased from Invitrogen Life Technologies (Carlsbad, CA, USA).

Animals and animal model. The present study was conducted in accordance with the Guide for the Local Care and Use of Laboratory Animals and was approved by the Ethics Committee of The Central Hospital of Wuhan (Wuhan, China). All surgery and subsequent analyses were performed in a blinded manner. Male BALA/c mice (18-25 g; 10-12 weeks) purchased from the Experimental Animal Center of Hubei Province (Wuhan, China), were randomized to undergo LAD ligation or sham surgery. The animals were anesthetized with an intraperitoneal injection of $1 \%$ pentobarbital sodium $(30 \mathrm{mg} / \mathrm{kg})$ and local injection of $1 \mathrm{ml} 1 \%$ lidocaine. Thoracotomy was performed via the third left intercostal space. The pericardium was opened and prolene (8-0) was used to ligate the LAD. Visible blanching and hypokinesis of the anterior wall of the left ventricle and swelling of the left atrium were indicative of successful ligation. Sham surgery consisted of the same procedure, however, the suture was passed through the myocardium beneath the LAD without ligation. Animals were divided into several groups. Group I mice received the sham surgery. Group II mice received LAD ligation. Group III mice received LAD ligation and normal saline and group IV mice received LAD ligation and lycopene $(10 \mathrm{mg} / \mathrm{kg} / \mathrm{day}$, intragastrically for 4 weeks).

Terminal deoxynucleotidyl transferase dUTP nick end labeling (TUNEL) staining. Hearts were perfused with $0.1 \mathrm{ml}$ phosphate-buffered saline (PBS) via the left coronary artery and then dissected and fixed in $4 \%$ paraformaldehyde in PBS ( $\mathrm{pH}$ 7.4) for $24 \mathrm{~h}$ at room temperature. Fixed tissues were embedded in paraffin and five sections of $6 \mathrm{~mm}$ thickness were cut from each tissue block. TUNEL staining was performed according to the manufacturer's instructions (Roche Diagnostics, Mannheim, Germany). Total nuclei were stained with DAPI (Vector Laboratories Inc., Burlingame, CA, USA). Additionally, cardiac tissue was specifically labeled with the mouse monoclonal $\alpha$-actinin antibody. (ab9465, Abcam). The apoptotic index (the number of positively stained nuclei / total number of nuclei counted x 100\%) was determined in a blinded manner.

Western blot analysis. Protein $(50 \mu \mathrm{g})$ was extracted from myocardial tissue and lysed in radioimmunoprecipitation assay lysis buffer (Cell Signaling Technology, Inc.) for use in SDS-PAGE (Invitrogen Life Technologies). The proteins were then transferred onto nitrocellulose membranes and inhibited with $5 \%$ nonfat dry milk in tris-buffered saline (TBS; Cell Signaling Technology, Inc.) for $90 \mathrm{~min}$ at room temperature. Membranes were probed with several primary antibodies against caspase-3, -8, -9, NF- $\mathrm{B}$ p65 and phospho-NF- $\kappa$ B p65 overnight. The next day, it was washed using 1X TBS with Tween-20 (TBST) and incubated for $1 \mathrm{~h}$ with horseradish peroxidase-labeled polyclonal mouse anti rabbit antibody $(1: 2,000)$ and anti-avidin antibodies $(1: 1,000)$ (Jackson Immunoresearch, West Grove, PA, USA) in double anti-TBST fluid. The membrane was washed three times and the film was placed in $10 \mathrm{ml}$ LumiGLO solution (Cell Signaling Technology, Inc.) for $1 \mathrm{~min}$. Following development, the images were put into an automatic image analyzer to determine the function of proteins and the reference gray scale values. Monoclonal GAPDH was used separately as a loading control.

Quantitative polymerase chain reaction ( $q P C R$ ) analysis. Total mRNA was extracted from the myocardial tissue using TRIzol according to the manufacturer's instructions and cDNA was synthesized using oligo (dT) primers with the Transcriptor First Strand cDNA synthesis kit (Roche Diagnostics). Selected gene differences were confirmed by qPCR using SYBR green (Roche Diagnostics) and results were normalized against GAPDH gene expression. The sequences of all primers used were as follows: Transforming growth factor- $\beta 1$ (TGF- $\beta 1$ ), forward 5'-AACAACGCCATCTATGAG-3' and reverse 5'-TATTCCGTCTCCTTGGTT-3'; collagen I, forward 5'-AGGCTTCAGTGGTTTGGATG-3' and reverse 5'-CACCAACAGCACCATCGTTA-3'; collagen III, forward 5'-CCCAACCCAGAGA TCCCATT-3' and reverse 5'-GAAGCACAGGAGCAGGTGTAGA-3'; TNF- $\alpha$, forward 5'-CATCT TCTCAAAATTCGAGTGACAA-3' and reverse 5'-TGGGAGTAGACAAGGTACAACCC-3'; IL-1 $\beta$, forward 5'-CCGTGGACCTTCCAGGATGA-3' and reverse 5'-GGGAACGTCACA CACCAGCA-3'; GAPDH, forward 5'-ACTCCACTCACGGCAAATTC-3' and reverse 5'-TCTC CATGGTGGTGAAGACA-3'.

Statistical analysis. Data are presented as the mean \pm standard deviation. Comparisons between four groups were performed using one-way analysis of variance. $\mathrm{P}<0.05$ was considered to indicate a statistically significant difference.

\section{Results}

Lycopene improves cardiac remodeling in MI mice. A model of MI was established by ligating the left coronary artery. Cardiac remodeling was estimated by detecting the mRNA expression of fibrosis markers, including TGF- $\beta 1$, collagen I 


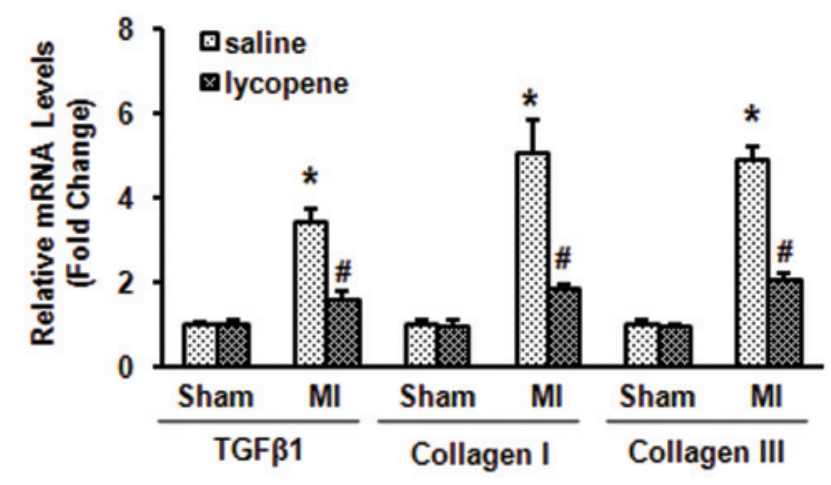

Figure 1. Expression levels of TGF- $\beta 1$, collagen I and collagen III. The mRNA levels of TGF- $\beta 1$, collagen I and collagen III in the ventricle of C57BL/6J wild type mice following sham or MI surgery with or without lycopene $(10 \mathrm{mg} / \mathrm{kg} /$ day $)$. Data are presented as the mean \pm standard deviation. $\mathrm{n}=6$ mice per experimental group. ${ }^{*} \mathrm{P}<0.05$, vs. saline + sham; ${ }^{\#} \mathrm{P}<0.05$, vs. saline + MI. TGF- $\beta 1$, transforming growth factor- $\beta 1$; MI, myocardial infarction.

and III. Compared with the MI group adminstered with saline, administration of lycopene $(10 \mathrm{mg} / \mathrm{kg} /$ day $)$ reduced the mRNA expression of TGF- $\beta 1$, collagen I and III ( $\mathrm{P}<0.05$; Fig. 1$)$. These results demonstrated that lycopene protected the mice from MI.

Lycopene reduces inflammatory responses in MI mice. The inflammatory cytokines TNF- $\alpha$ and IL- $1 \beta$ were increased in mice 4 weeks after MI induction by LAD ligation. However, treatment with $10 \mathrm{mg} / \mathrm{kg} /$ day lycopene significantly reduced the mRNA expression of TNF- $\alpha$ and IL- $1 \beta$ in infarcted myocardium compared with the normal saline-treated group $(\mathrm{P}<0.05$; Fig. 2). These results suggested that lycopene exerted an anti-inflammatory effect on MI mice.

Lycopene attenuates cardiomyocyte apoptosis in MI mice. A marked decrease in TUNEL-positive nuclear staining was observed in the sectioned left ventricular myocardium following treatment with lycopene $(10 \mathrm{mg} / \mathrm{kg} /$ day $)$ compared with saline at 4 weeks post-MI $(\mathrm{P}<0.05$; Fig. $3 \mathrm{~A})$. TUNEL-positive nuclear staining was not observed in the sham group. The level of apoptosis, assessed by detecting the protein expression of cleaved caspase- $3,-8$ and -9 was significantly higher 4 weeks post-MI compared with the sham group. However, the expression of caspase- $3,-8$ and -9 were markedly decreased following treatment with lycopene $(10 \mathrm{mg} / \mathrm{kg} / \mathrm{day})$ compared with saline $(\mathrm{P}<0.05$; Fig. 3B and C). These data indicated that lycopene inhibited myocardial cell apoptosis in mice subjected to MI.

Lycopene inhibits activation of the NF- $\kappa B$ signaling pathway in MI mice. In order to identify the mechanism involved in the inhibition of the inflammatory response and cell apoptosis by lycopene in MI mice, the activation of NF- $\mathrm{KB}$ in the ischemic myocardium was detected. Western blot analysis of nuclear and cytoplasmic extracts confirmed that $10 \mathrm{mg} / \mathrm{kg} /$ day lycopene decreased the nuclear localization of NF- $\mathrm{KB}$ p65 4 weeks post-MI (Fig. 4A and B). In the ischemic myocardium, the level of NF-kB p65 phosphorylation was markedly induced 4 weeks post-MI. NF- $\mathrm{kB}$ p 65 phosphorylation was reduced in the lycopene treatment group compared with the MI group administered with saline (Fig. 4A and B). These results suggest that lycopene

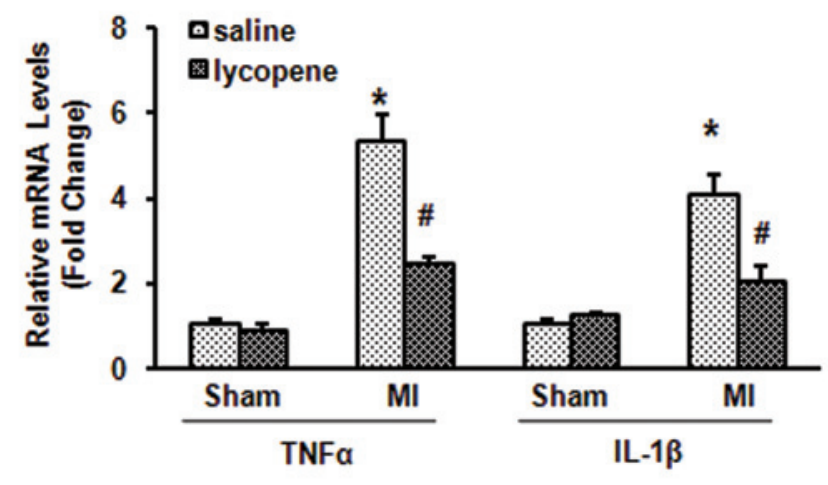

Figure 2. Expression levels of TNF- $\alpha$ and IL-1 $1 \beta$. The mRNA levels of TNF- $\alpha$ and IL- $1 \beta$ in the ventricle of C57BL/6J wild type mice following sham or MI surgery with or without lycopene $(10 \mathrm{mg} / \mathrm{kg} / \mathrm{day})$. Data are presented as the mean \pm standard deviation. $\mathrm{n}=6$ mice per experimental group, ${ }^{*} \mathrm{P}<0.05$, vs. saline + sham; ${ }^{\prime} \mathrm{P}<0.05$, vs. saline + MI. TNF- $\alpha$, tumor necrosis factor- $\alpha$; IL-1 $\beta$, interleukin-1 $\beta$; MI, myocardial infarction.

may exert an anti-inflammatory and anti-apoptotic effect by suppressing NF- $\mathrm{kB}$ activation in MI mice.

\section{Discussion}

Coronary heart disease is expected to become the leading worldwide cause of mortality by 2020 (22). The primary pathological manifestation of coronary artery disease is myocardial damage, which most likely develops into cardiac remodeling. Cardiac remodeling post-MI involves a complex set of events that paradoxically causes an increase in tissue injury characterized by inflammation, apoptosis, infarction and fibrosis (23-25). MI causes inflammatory cell infiltration into the infarct zone and increases the production of proinflammatory cytokines (26). Therefore, inhibition of inflammatory cell infiltration could reduce cardiac damage and attenuate the development of MI. Lycopene could be useful in treating various inflammatory diseases (27-29). The results of the present study indicated that lycopene offers protection from myocardial cell injury and reduces the production of proinflammatory cytokines, including TNF- $\alpha$ and IL- $1 \beta$ in infarcted myocardium.

Cardiac apoptosis is a key pathological feature of MI $(30,31)$. Two major pathways that lead to apoptosis have been identified, including the mitochondrial pathway and the death-receptor pathway $(32,33)$. These pathways lead to the activation of caspase- $3,-8$ and -9 , the central mediators of the cascade (34). Cascade protein levels are increased in myocardial samples from patients with right ventricular dysplasia and heart failure $(35,36)$. In mice, cardiomyocyte apoptosis may occur transiently during the first few days following MI in the ischemic area, whereas left ventricular remodeling following MI is associated with apoptosis in the myocardium remote from the infarct. Previous studies have also demonstrated that the amplification of apoptosis was sufficient to increase infarct size, indicating a critical role for cardiomyocyte apoptosis in myocardial infarct expansion $(37,38)$. The present study demonstrated that myocardial cell apoptosis, measured via TUNEL assay and caspase- $3,-8$ and -9 protein expression levels, was inhibited by lycopene treatment, implying an antiapoptotic role for lycopene and correlating with protection from myocardial cell injury. 


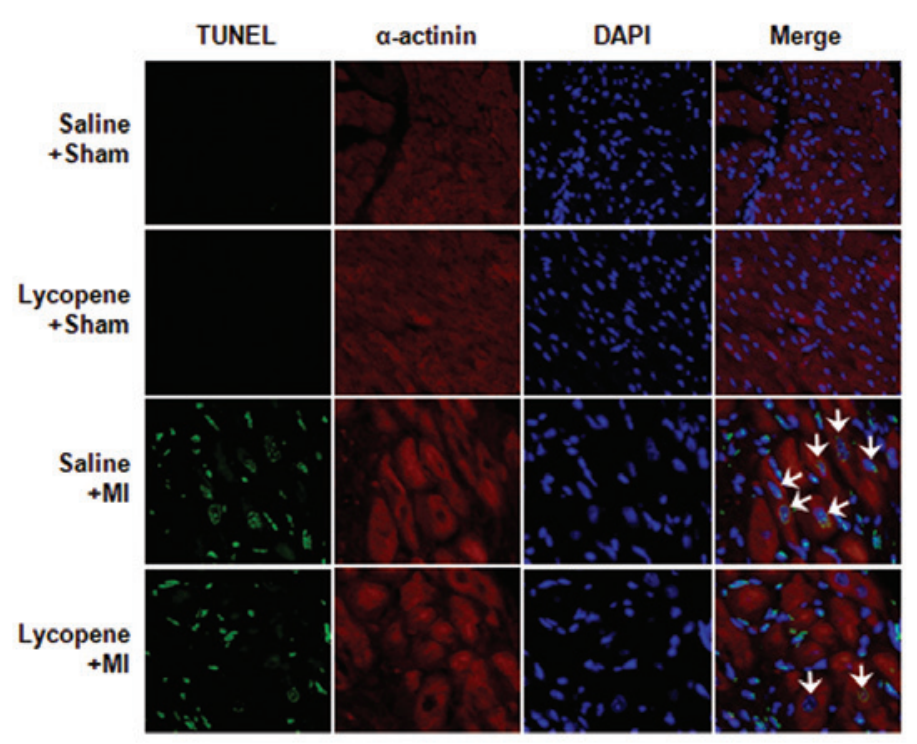

B

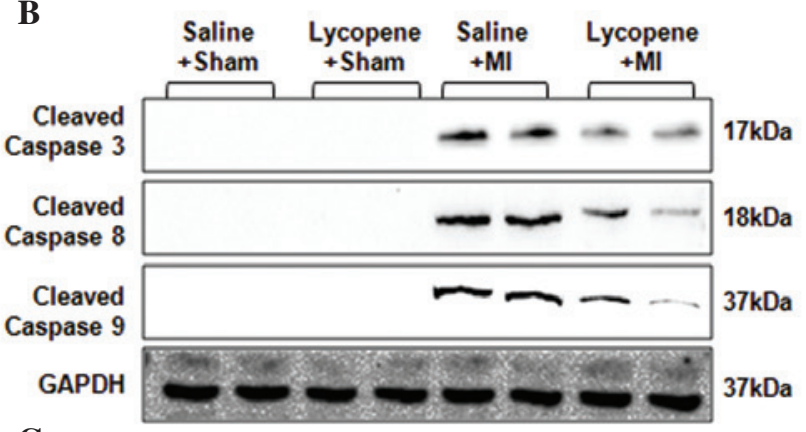

C

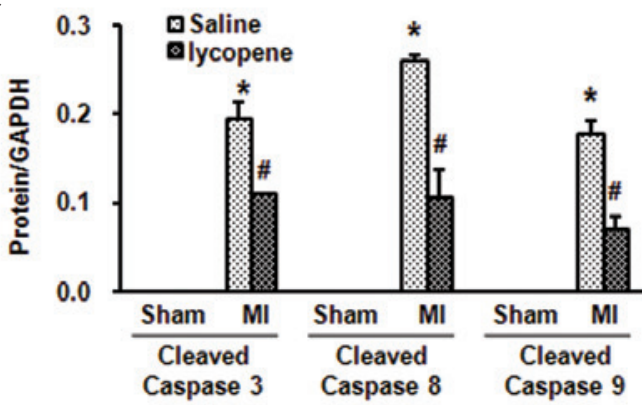

Figure 3. Effects of lycopene on cardiomyocyte apoptosis. (A) Representative images of TUNEL staining in heart sections of C57BL/6J wild type mice following sham or MI surgery with or without lycopene (10 mg/kg/day). (Green: TUNEL; Red: $\alpha$-actinin; Blue: DAPI). (B and C). Expression of cleaved-caspase-3, -8 and -9 protein in samples from the ventricle of C57BL/6J wild type mice following sham or MI surgery with or without lycopene (10mg/kg/day). (B) Representative western blotting; (C) Quantitative results. GAPDH was used as the loading control. Data are presented as mean \pm standard deviation. At least three independent experiments were performed. ${ }^{~} \mathrm{P}<0.05$, vs. saline + sham; ${ }^{*} \mathrm{P}<0.05$, vs. saline $+\mathrm{MI}$. TUNEL, terminal deoxynucleotidyl transferase dUTP nick end labeling; GADPH, glyceraldehyde 3-phosphate dehydrogenase; MI, myocardial infarction.

A

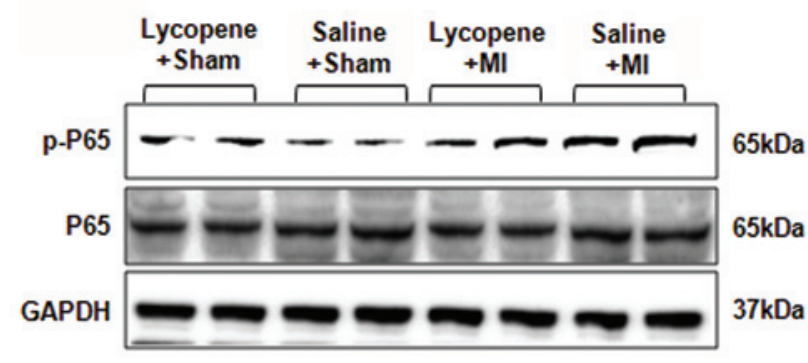

B

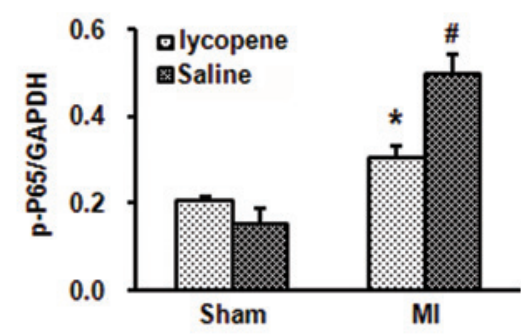

Figure 4. Effects of lycopene on p65 signaling. (A and B) Expression of p-P65 and P65 protein in samples from the ventricle of C57BL/6J wild type mice following sham or MI surgery with or without lycopene (10 mg/kg/day). (A) Representative western blotting; (B) Quantitative results. GAPDH was used as the loading control. Data are presented as the mean \pm standard deviation. At least three independent experiments were performed. $\mathrm{P}<0.05$, vs. saline + sham; ${ }^{\#} \mathrm{P}<0.05$, vs. saline + MI. GADPH, glyceraldehyde 3-phosphate dehydrogenase; MI, myocardial infarction.

Therefore, it was hypothesized that lycopene treatment may reduce ventricular remodeling by decreasing cardiomyocyte apoptosis in the infarct and peri-infarct regions.

In addition, lycopene decreased the nuclear localization of nuclear $\mathrm{NF}-\kappa \mathrm{B}$ p 65 and markedly suppressed NF- $\kappa \mathrm{B}$ activation. $N F-\kappa B$ is a transcription factor that regulates several inflammatory genes and is a key regulator of the inflammatory response (39). Activation of $\mathrm{NF}-\kappa \mathrm{B}$ induces the transcription of pro-inflammatory genes, including TNF- $\alpha$ and IL-1 $\beta$. It has been demonstrated that NF- $\kappa \mathrm{B}$ is activated by myocardial injury, with cardiac myocytes and interstitial cells being important sources of NF- $\kappa \mathrm{B}(40,41)$. Proinflammatory cytokines exert their actions on target cells by transactivating NF- $\kappa \mathrm{B}$, thus cells, including cardiomyocytes, respond to proinflammatory cytokines by activating $\mathrm{NF}-\kappa \mathrm{B}$. The results of the present study suggest that the cardioprotective effect of lycopene may be due to reduced production of proinflammatory cytokines via inhibition of the $\mathrm{NF}-\kappa \mathrm{B}$ activity induced by local myocardial injury. Therefore, this suggests that lycopene may have a cardioprotective effect associated with the inhibition of local myocardial inflammation and apoptosis.

The present study provided evidence of a novel cardioprotective effect of lycopene in a mouse model of MI. This protection was conferred, at least partially, by its anti-inflammatory and antiapoptotic actions following myocardial injury. These findings may provide a basis for a new therapeutic application of lycopene in the prevention of myocardial cell injury following MI. 


\section{Acknowledgements}

The present study was supported by grants from the National Natural Science Foundation of China (no. 81300214).

\section{References}

1. Sutton MG and Sharpe N: Left ventricular remodeling after myocardial infarction: pathophysiology and therapy. Circulation 101: 2981-2988, 2000

2. Frangogiannis NG, Smith CW and Entman ML: The inflammatory response in myocardial infarction. Cardiovasc Res 53: 31-47, 2002.

3. Dorn GW 2nd: Apoptotic and non-apoptotic programmed cardiomyocyte death in ventricular remodelling. Cardiovasc Res 1: 465-473, 2009.

4. Frantz S, Bauersachs J and Ertl G: Post-infarct remodelling: contribution of wound healing and inflammation. Cardiovasc Res 81: 474-481, 2009.

5. Nian M, Lee $P$, Khaper $N$ and Liu P: Inflammatory cytokines and postmyocardial infarction remodeling. Circ Res 94: 1543-1553, 2004

6. Koyama M, Kelly TR and Watanabe KA: Novel type of potential anticancer agents derived from chrysophanol and emodin. Some structure-activity relationship studies. J Med Chem 31: 283-284, 1988.

7. Sun M, Dawood F, Wen WH, Chen M, Dixon I, Kirshenbaum LA and Liu PP: Excessive tumor necrosis factor activation after infarction contributes to susceptibility of myocardial rupture and left ventricular dysfunction. Circulation 110: 3221-3228, 2004.

8. Baldi A, Abbate A, Bussani R, Patti G, Melfi R, Angelini A, Dobrina A, Rossiello R, Silvestri F, Baldi F and Di Sciascio G: Apoptosis and post-infarction left ventricular remodeling. J Mol Cell Cardiol 34: 165-174, 2002.

9. Palojoki E, Saraste A, Eriksson A, Pulkki K, Kallajoki M, Voipio-Pulkki LM and Tikkanen I: Cardiomyocyte apoptosis and ventricular remodeling after myocardial infarction in rats Am J Physiol Heart Circ Physiol 280: H2726-H2731, 2001.

10. Yeh $\mathrm{CH}$, Chen $\mathrm{TP}, \mathrm{Wu} \mathrm{YC}$, Lin YM and Jing Lin P: Inhibition of NFkappaB activation with curcumin attenuates plasma inflammatory cytokines surge and cardiomyocytic apoptosis following cardiac ischemia/reperfusion. J Surg Res 125: 109-116, 2005.

11. Yeh CH, Chen TP, Lee CH, Wu YC, Lin YM and Lin PJ: Cardiomyocytic apoptosis following global cardiac ischemia and reperfusion can be attenuated by peroxisome proliferator-activated receptor alpha but not gamma activators. Shock 26: 262-270, 2006.

12. Wang M, Tsai BM, Reiger KM, Brown JW and Meldrum DR: 17-beta-Estradiol decreases p38 MAPK-mediated myocardial inflammation and dysfunction following acute ischemia. J Mol Cell Cardiol 40: 202-212, 2006.

13. Kopp EB and Ghosh S: NF-kappa B and rel proteins in innate immunity. Adv Immunol 58: 1-27, 1995.

14. Kanno T, Franzoso G and Siebenlist U: Human T-cell leukemia virus type I Tax-protein-mediated activation of NF-kappa B from p100 (NF-kappa B2)-inhibited cytoplasmic reservoirs. Proc Natl Acad Sci USA 91: 12634-12638, 1994.

15. Onai Y, Suzuki J, Maejima Y, Haraguchi G, Muto S, Itai A and Isobe M: Inhibition of NF-\{kappa\}B improves left ventricular remodeling and cardiac dysfunction after myocardial infarction. Am J Physiol Heart Circ Physiol 292: H530-H538, 2007.

16. Karppi J, Kurl S, Nurmi T, Rissanen TH, Pukkala E and Nyyssönen K: Serum lycopene and the risk of cancer: the Kuopio Ischaemic Heart Disease Risk Factor (KIHD) study. Ann Epidemiol 19: 512-518, 2009.

17. Riccioni G, Mancini B, Di Ilio E, Bucciarelli T and D'Orazio N: Protective effect of lycopene in cardiovascular disease. Eur Rev Med Pharmacol Sci 12: 183-190, 2008.

18. Parvin $\mathrm{R}$ and Akhter $\mathrm{N}$ : Protective effect of tomato against adrenaline-induced myocardial infarction in rats. Bangladesh Med Res Counc Bull 34: 104-108, 2008.

19. Upaganlawar A, Gandhi $H$ and Balaraman R: Effect of vitamin $E$ alone and in combination with lycopene on biochemical and histopathological alterations in isoproterenol-induced myocardial infarction in rats. J Pharmacol Pharmacother 1: 24-31, 2010.

20. Simone RE, Russo M, Catalano A, Monego G, Froehlich K, Boehm V and Palozza P: Lycopene inhibits NF- $\kappa$ B-mediated IL-8 expression and changes redox and PPAR $\gamma$ signalling in cigarette smoke-stimulated macrophages. PLoS One 6: e19652, 2011.
21. Gouranton E, Thabuis C, Riollet C, Malezet-Desmoulins C, El Yazidi C, Amiot MJ, Borel P and Landrier JF: Lycopene inhibits proinflammatory cytokine and chemokine expression in adipose tissue. J Nutr Biochem 22: 642-648, 2011.

22. Rajadurai M and Stanely Mainzen Prince P: Preventive effect of naringin on cardiac markers, electrocardiographic patterns and lysosomal hydrolases in normal and isoproterenol-induced myocardial infarction in Wistar rats. Toxicology 230: 178-188, 2007.

23. Lujan HL and DiCarlo SE: Mimicking the endogenous current of injury improves post-infarct cardiac remodeling. Med Hypotheses 81: 521-523, 2013.

24. Meloni M, Marchetti M, Garner K, Littlejohns B, Sala-Newby G, Xenophontos N, Floris I, Suleiman MS, Madeddu P, Caporali A and Emanueli C: Local inhibition of microRNA-24 improves reparative angiogenesis and left ventricle remodeling and function in mice with myocardial infarction. Mol Ther 21: 1390-1402, 2013.

25. Kanno Y, Watanabe R, Zempo H, Ogawa M, Suzuki J and Isobe M: Chlorogenic acid attenuates ventricular remodeling after myocardial infarction in mice. Int Heart J 54: 176-180, 2013.

26. Lei J, Xue S, Wu W, Zhou S, Zhang Y, Yuan G and Wang J: Sdcl overexpression inhibits the p38 MAPK pathway and lessens fibrotic ventricular remodeling in MI rats. Inflammation 36: 603-615, 2013

27. Morgia G, Cimino S, Favilla V, Russo GI, Squadrito F, Mucciardi G, Masieri L, Minutoli L, Grosso G and Castelli T: Effects of Serenoa repens, selenium and lycopene (Profluss ${ }^{\circledR}$ ) on chronic inflammation associated with benign prostatic hyperplasia: results of 'FLOG' (Flogosis and Profluss in Prostatic and Genital Disease), a multicentre Italian study. Int Braz J Urol 39: 214-221, 2013.

28. Renju GL, Muraleedhara Kurup G and Saritha Kumari CH: Anti-inflammatory activity of lycopene isolated from Chlorella marina on type II collagen induced arthritis in Sprague Dawley rats. Immunopharmacol Immunotoxicol 35 : 282-291, 2013.

29. Hadad N and Levy R: The synergistic anti-inflammatory effects of lycopene, lutein, $\beta$-carotene and carnosic acid combinations via redox-based inhibition of NF- $\mathrm{B}$ signaling. Free Radic Biol Med 53: 1381-1391, 2012.

30. Abbate A and Narula J: Role of apoptosis in adverse ventricular remodeling. Heart Fail Clin 8: 79-86, 2012.

31. Singh SS and Kang PM: Mechanisms and inhibitors of apoptosis in cardiovascular diseases. Curr Pharm Des 17: 1783-1793, 2011.

32. Sinha K, Das J, Pal PB and Sil PC: Oxidative stress: the mitochondria-dependent and mitochondria-independent pathways of apoptosis. Arch Toxicol 87: 1157-1180, 2013.

33. Delmas D, Solary E and Latruffe N: Resveratrol, a phytochemical inducer of multiple cell death pathways: apoptosis, autophagy and mitotic catastrophe. Curr Med Chem 18: 1100-1121, 2011.

34. MacKenzie SH and Clark AC: Death by caspase dimerization. Adv Exp Med Biol 747: 55-73, 2012.

35. Moorjani N, Westaby S, Narula J, Catarino PA, Brittin R, Kemp TJ, Narula N and Sugden PH: Effects of left ventricular volume overload on mitochondrial and death-receptor-mediated apoptotic pathways in the transition to heart failure. Am J Cardiol 103: 1261-1268, 2009.

36. Satoh M, Minami Y, Takahashi Y and Nakamura M: Immune modulation: role of the inflammatory cytokine cascade in the failing human heart. Curr Heart Fail Rep 5: 69-74, 2008.

37. Gao Y, Li T, Wu C, Bittle GJ, Chen S, Wu ZJ and Griffith BP: Pim-1 mediated signaling during the process of cardiac remodeling following myocardial infarction in ovine hearts. J Mol Cell Cardiol 63: 89-97, 2013.

38. Echtermeyer F, Harendza T, Hubrich S, Lorenz A, Herzog C, Mueller M, Schmitz M, Grund A, Larmann J, Stypmann J, Schieffer B, Lichtinghagen R, Hilfiker-Kleiner D, Wollert KC, Heineke J and Theilmeier G: Syndecan-4 signalling inhibits apoptosis and controls NFAT activity during myocardial damage and remodelling. Cardiovasc Res 92: 123-131, 2011.

39. Hoesel B and Schmid JA: The complexity of NF- $\kappa$ B signaling in inflammation and cancer. Mol Cancer 12: 86, 2013.

40. González A, Ravassa S, Beaumont J, López B and Díez J: New targets to treat the structural remodeling of the myocardium. J Am Coll Cardiol 58: 1833-1843, 2011.

41. Gordon JW, Shaw JA and Kirshenbaum LA: Multiple facets

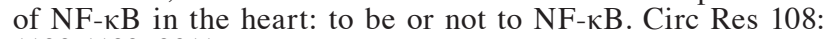
1122-1132, 2011. 